\title{
Nosocomial infections in a neonatal intensive care unit during 16 years: 1997-2012
}

\author{
Jane Eire Urzedo ${ }^{[1]}$, Maria Margarida Morena Domingos Levenhagen ${ }^{[1]}$, \\ Reginaldo Santos Pedroso ${ }^{[2]}$, Vânia Olivetti Steffen Abdallah ${ }^{[1]}$, \\ Sebastiana Silva Sabino ${ }^{[3]}$ and Denise Von Dolinger Brito ${ }^{[4]}$
}

[1]. Faculdade de Medicina, Universidade Federal de Uberlândia, Uberlândia, MG. [2]. Escola Técnica de Saúde, Universidade Federal de Uberlândia, Uberlândia, MG. [3]. Hospital de Clínicas, Universidade Federal de Uberlândia, Uberlândia, MG. [4]. Instituto de Ciências Biomédicas, Universidade Federal de Uberlândia, Uberlândia, MG.

\begin{abstract}
Introduction: Surveillance of nosocomial infections (NIs) is an essential part of quality patient care; however, there are few reports of National Healthcare Safety Network (NHSN) surveillance in neonatal intensive care units (NICUs) and none in developing countries. The purpose of this study was to report the incidence of NIs, causative organisms, and antimicrobial susceptibility patterns in a large cohort of neonates admitted to the NICU during a 16-year period. Methods: The patients were followed 5 times per week from birth to discharge or death, and epidemiological surveillance was conducted according to the NHSN. Results: From January 1997 to December 2012, 4,615 neonates, representing 62,412 patient-days, were admitted to the NICU. The deviceassociated infection rates were as follows: 17.3 primary bloodstream infections per 1,000 central line-days and 3.2 pneumonia infections per 1,000 ventilator-days. A total of 1,182 microorganisms were isolated from sterile body site cultures in 902 neonates. Coagulase-negative staphylococci (CoNS) (34.3\%) and Staphylococcus aureus $(15.6 \%)$ were the most common etiologic agents isolated from cultures. The incidences of oxacillin-resistant CoNS and Staphylococcus aureus were $86.4 \%$ and $28.3 \%$, respectively. Conclusions: The most important NI remains bloodstream infection with staphylococci as the predominant pathogens, observed at much higher rates than those reported in the literature. Multiresistant microorganisms, especially oxacillin-resistant staphylococci and gram-negative bacilli resistant to cephalosporin were frequently found. Furthermore, by promoting strict hygiene measures and meticulous care of the infected infants, the process itself of evaluating the causative organisms was valuable.
\end{abstract}

Keywords: Nosocomial infection. Surveillance system. Critical neonates.

\section{INTRODUCTION}

Newborns admitted to intensive care units (ICUs) are at high risk for developing nosocomial infections (NIs) because of the severity of their illness and exposure to invasive medical devices such as mechanical ventilators and central venous catheters (CVCs) and resistant microorganisms ${ }^{1,2}$.

Surveillance of NIs is an essential part of quality patient care; however, there are few reports of National Healthcare Safety Network (NHSN) surveillance in neonatal intensive care units (NICUs) and none in developing countries ${ }^{3}$. The objective of this study was to report the incidence of NIs, causative organisms, and antimicrobial susceptibility patterns in a large cohort of neonates admitted to the NICU during a 16 -year period.

Address to: Dr $^{\mathrm{a}}$ Denise Von Dolinger de Brito. ICBIM/UFU. Av. Pará 1720, 38400-902 Uberlândia, MG, Brasil.

Phone: 5534 3218-2236

e-mail: denisevondolinger@gmail.com

Received 6 May 2014

Accepted 24 June 2014

\section{METHODS}

The Medical Hospital of the Federal University of Uberlândia is a 533-bed public teaching general hospital and a tertiary care center. The NICU consists of 2 rooms with a capacity of 15 neonates and also serves as a referral center for several hospitals in the vicinity.

From January 1997 to December 2012, all neonates admitted for more than $24 \mathrm{~h}$ were included. Participation in the study was voluntary and confidential. The patients included were followed 5 times per week from birth to discharge or death. Epidemiological surveillance was conducted according to the National Nosocomial Infections Surveillance System ${ }^{4}$ and

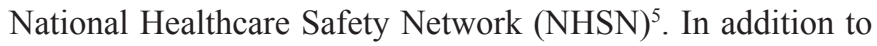
standard hospital epidemiological measures, we also analyzed data adjusted for the duration of exposure to the hospital environment or to invasive devices (reported as patient-days and device-days, respectively). The neonates were stratified into the following birth weight categories: $\leq 750 ; 751-1,000$; $1,001-1,500 ; 1,501-2,500$ and $>2,500 \mathrm{~g}$.

An nosocomial infection was defined as an infection not present or incubating at the time of NICU admission, with onset after $48 \mathrm{~h}$ of stay. Mechanical ventilator-associated pneumonia (VAP) was 
defined as the development of pneumonia in a neonate $48 \mathrm{~h}$ or more after initiation of mechanical ventilation. Primary bloodstream infection (pBSI) was defined as laboratoryconfirmed bloodstream infection (BSI) or clinical sepsis not related to infection at another site, except for an intravascular device. A central venous catheter-related pBSI was defined as the presence of all of the following criteria: I) bacteremia or fungemia in a neonate with a CVC with at least 1 positive blood culture obtained from a peripheral vein; II) clinical manifestations of infection (i.e., fever $\left[>38^{\circ} \mathrm{C}\right]$, hypothermia $\left[<37^{\circ} \mathrm{C}\right]$, apnea, or bradycardia) and no apparent source for the BSI except the CVC; and III) a positive semiquantitative ( $>15$ colony-forming units $[\mathrm{CFU}] /$ catheter segment) or quantitative $\left(>10^{3} \mathrm{CFU} /\right.$ catheter segment) culture from which the same organism (species and antibiogram) was isolated from the catheter segment and peripheral blood. Umbilical catheters were considered central lines ${ }^{6}$.

In accordance with institutional protocols, the same initial diagnostic work-up was applied to all neonates upon suspicion of pBSI or VAP, irrespective of the clinical condition. This included a plan of clinical assessment and care concerning thermoregulation, ventilation, circulation, metabolism, and nutrition. All infants underwent a complete physical examination, continuous electrocardiography, pulse oximetry, and central or peripheral venous catheterization as needed. A chest radiograph was performed, and blood samples were drawn for arterial gasometry; a complete hemogram; and sodium, potassium, chlorine, glucose, and C-reactive protein measurement. Two blood cultures were drawn on separate occasions before initiation of antimicrobial treatment in all neonates who had suspected pBSI or VAP, except for extremely low birth weight neonates or neonates who had fulminant sepsis, in whom antimicrobial treatment was initiated immediately after a single blood culture was drawn. Cultures for anaerobic bacteria were not routinely obtained.

Blood cultures that were positive for organisms that generally are considered to be contaminants (e.g., Corynebacterium sp., Bacillus sp., micrococci, and diphtheroids) were excluded from analysis. The coagulase-negative staphylococci (CoNS) were considered true pathogens when 2 blood cultures drawn on separate occasions were positive for the same species and antibiogram and the organism was not related to infection at another site or when 1 blood culture was positive in a patient with an intravascular access device and the physician instituted appropriate antimicrobial treatment. For all other pathogens, a single positive blood culture was considered significant.

Specimens for culture were collected based on clinical criteria established by the medical staff. Microbiological data were obtained from the Uberlândia University Hospital Microbiology Laboratory.

\section{Ethical considerations}

Ethical approved was obtained from the Uberlândia Federal University Ethics Committee.

\section{RESULTS}

From January 1997 to December 2012, 4,615 neonates were admitted to the NICU, and 192 months of data representing 62,412 patient-days were available for analysis.

The average weight of the neonates was $1,830.6 \mathrm{~g}$, with the highest proportion $(34 \%)$ of neonates in the weight category $>2,500 \mathrm{~g}$. The proportion of neonates with a gestational age $>31$ weeks was $55.3 \%$. The average length of stay in the unit was 14.9 days. The invasive device use rate [number of device-days/ number of patient-days (times) 100] was 0.8 for CVC and 0.4 for mechanical ventilation. The device-associated infection rates were $17.3 \mathrm{pBSIs}$ per 1,000 central line-days and 3.2 pneumonia infections per 1,000 ventilator-days. The mortality rate in neonates with NI was $10.4 \%$, and overall mortality was 9.1\% (Table 1).

In total, 902 (19\%) infants developed an NI, and 194 (21.5\%) of these had more than 1 infection for a total of 1,096 NIs, principally BSI $(752 ; 68.6 \%)$ and conjunctivitis $(208 ; 19 \%)$ (Table 2).

A total of 1,182 microorganisms were isolated from sterile body site cultures in 902 neonates. Gram-positive bacteria and gram-negative bacteria accounted for $636(53.8 \%)$ and $460(38.9 \%)$ isolates, respectively. Fungi accounted for the remaining $86(7.3 \%)$ isolates. The distribution of microorganisms isolated according to year is shown in Figure 1.

In a comparative analysis of infection episodes occurring during the 16-year study period, we observed a reduction in the years 2004 and 2005 owing to a retirement unit and stricter hand hygiene and infection control measures. The distribution of episodes according to year is shown in Figure 2.

CoNS (34.3\%) and Staphylococcus aureus (15.6\%) were the most common etiologic agents isolated from cultures. Selected antimicrobial susceptibility patterns are presented in Table 3, showing that $86.4 \%$ of CoNS and $28.3 \%$ of Staphylococcus aureus were oxacillin-resistant. Of special interest is the resistance of Serratia marcescens, Klebsiella pneumoniae, and Pseudomonas aeruginosa to third-generation cephalosporin, the first 2 in BSI cases and the last in conjunctivitis cases.

\section{DISCUSSION}

Analysis of our data suggests that participation in surveillance systems, like the NHSN, is important for comparing individual NICU data with other data from the literature. However, comparing surveillance data from different countries is not straightforward. For example, device use rates vary widely between different countries, suggesting differences in either illness severity or clinical practice.

There is little information in the literature regarding the usefulness of assessing infection rates according to patientdays or device-days, that is, standard hospital epidemiological measures adjusted for the duration of exposure to the hospital environment or to invasive devices, such as 
TABLE 1 - Patient characteristics, device use, and number of nosocomial infections.

\begin{tabular}{|c|c|c|c|c|c|}
\hline & \multirow[b]{2}{*}{ Total } & \multicolumn{4}{|c|}{ Year of participation } \\
\hline & & $1997-2000$ & $2001-2004$ & $2005-2008$ & 2009-2012 \\
\hline Neonates (n) & 4,615 & 1,150 & 1,201 & 1,108 & 1,156 \\
\hline Pooled mean birth weight (g) & $1,830.6$ & $1,562.4$ & $1,488.0$ & $2,289.5$ & $1,982.6$ \\
\hline \multicolumn{6}{|c|}{ Neonates by birth weight class (\%) } \\
\hline$\leq 750 \mathrm{~g}$ & 5.4 & 6.0 & 4.6 & 5.8 & 5.2 \\
\hline $751-1,000 \mathrm{~g}$ & 10.7 & 12.4 & 8.9 & 10.2 & 11.1 \\
\hline $1,001-1,500 \mathrm{~g}$ & 20.8 & 24.9 & 19.8 & 20.3 & 18.2 \\
\hline $1,501-2,500 \mathrm{~g}$ & 29.1 & 29.1 & 28.9 & 30.4 & 27.9 \\
\hline$>2,500 \mathrm{~g}$ & 34.0 & 27.6 & 37.8 & 33.3 & 37.6 \\
\hline Patient-days (n) & 62,412 & 15,603 & 14,589 & 16,329 & 15,891 \\
\hline \multicolumn{6}{|c|}{ Neonates by gestational weeks (\%) } \\
\hline up to week 26 & 6.2 & 6.5 & 4.2 & 6.8 & 7.1 \\
\hline weeks $27-28$ & 12.7 & 14.1 & 10.5 & 12.9 & 13.2 \\
\hline weeks 29-30 & 25.8 & 22.9 & 18.2 & 30.2 & 31.9 \\
\hline week 31 and beyond & 55.3 & 56.5 & 67.1 & 50.1 & 47.8 \\
\hline Neonates who died (\%) & 9.1 & 9.6 & 9.3 & 8.4 & 9.1 \\
\hline \multicolumn{6}{|c|}{ device-use rates $($ device-days/patient-days $\times 100)$} \\
\hline $\mathrm{CVC}$ & 0.8 & 0.7 & 0.7 & 0.6 & 0.8 \\
\hline mechanical ventilator & 0.4 & 0.4 & 0.5 & 0.4 & 0.3 \\
\hline \multicolumn{6}{|l|}{ Neonates with NIs (\%) } \\
\hline BSI & 68.6 & 72.9 & 64.3 & 65.8 & 69.7 \\
\hline conjunctivitis & 19.0 & 17.5 & 20.6 & 19.6 & 19.1 \\
\hline pneumonia & 3.1 & 3.1 & 2.9 & 3.5 & 2.9 \\
\hline \multicolumn{6}{|l|}{ NIs/device-days $\times 1,000$} \\
\hline BSI (CVC-associated) & 17.3 & 12.7 & 19.1 & 18.4 & 19.1 \\
\hline pneumonia (VAP) & 3.2 & 3.1 & 2.9 & 3.5 & 3.1 \\
\hline
\end{tabular}

CVC: central venous catheter; BSI: bloodstream infection; NIs: nosocomial infections; VAP: ventilator-associated pneumonia.

TABLE 2 - Distribution of nosocomial infections.

\begin{tabular}{|c|c|c|}
\hline Infection & Number & Percentage \\
\hline Conjunctivitis & 208 & 19.0 \\
\hline Urinary tract infection & 52 & 4.8 \\
\hline Pneumonia & 34 & 3.1 \\
\hline Meningitidis & 12 & 1.1 \\
\hline Omphalitis & 10 & 0.9 \\
\hline Surgical wound & 7 & 0.6 \\
\hline Total & \multicolumn{2}{|c|}{1,096} \\
\hline
\end{tabular}




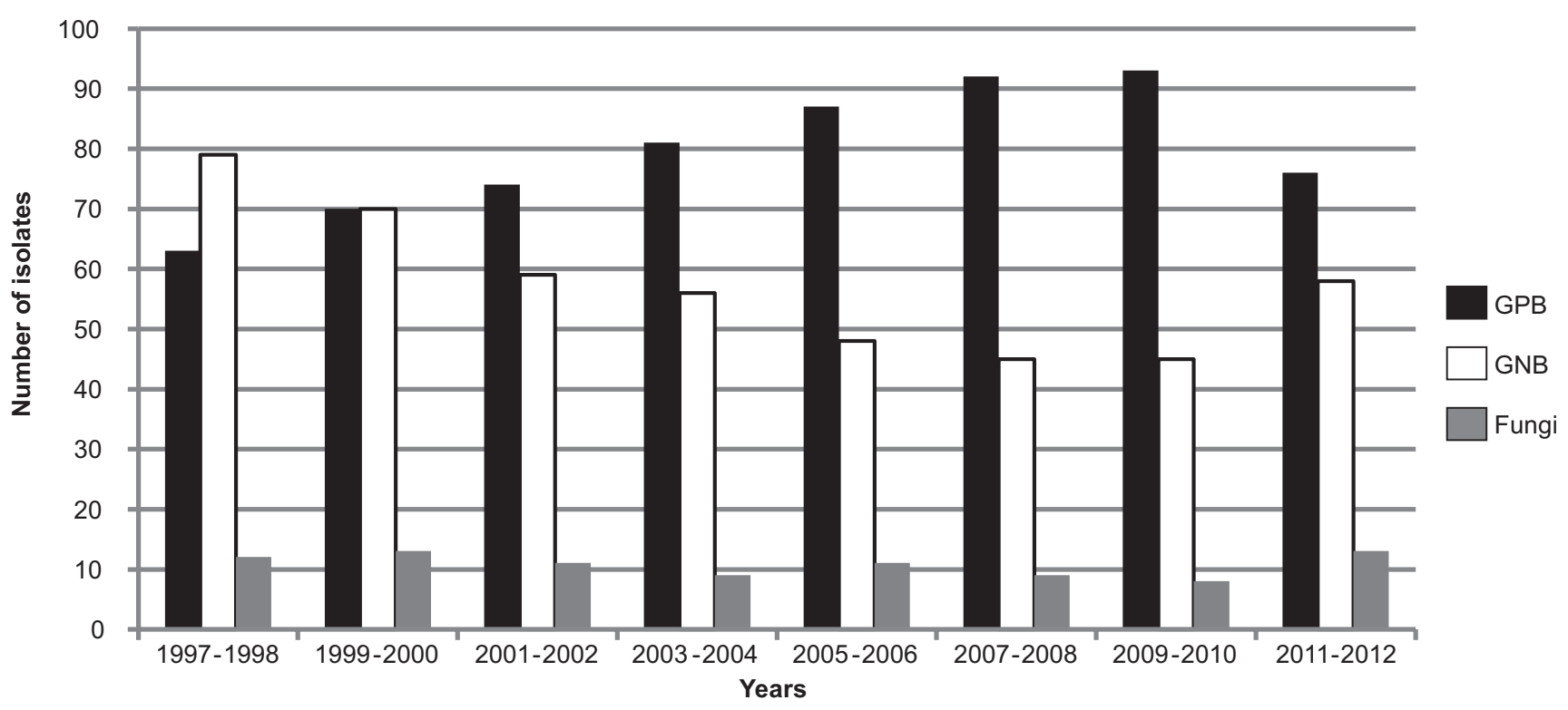

FIGURE 1 - Distribution of microorganisms in 2-year intervals.

GPB: gram-positive bacteria; GNB: gram-negative bacteria.

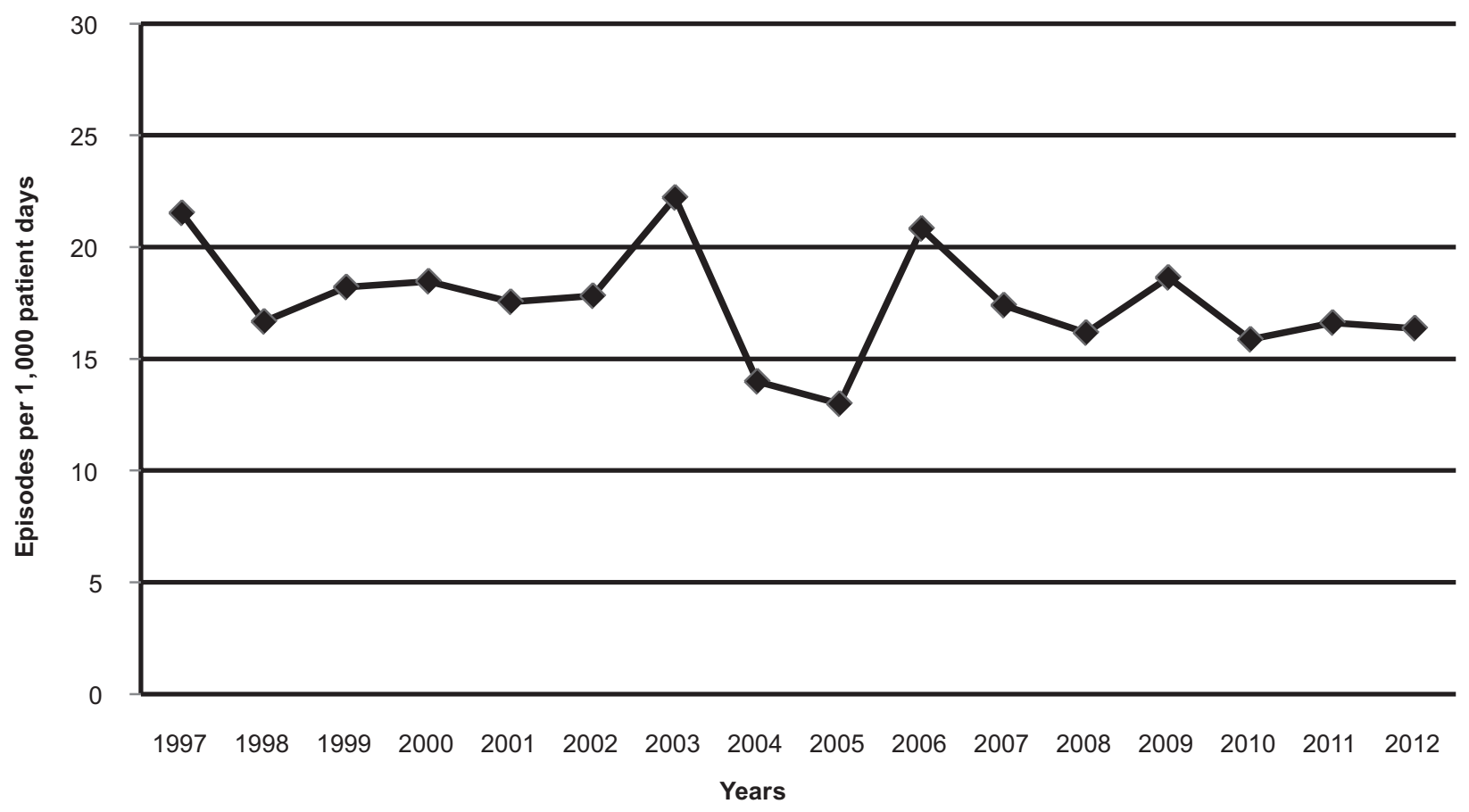

FIGURE 2 - Distribution of episodes per 1,000 patient-days during 1997-2012.

intravascular catheters or mechanical ventilation, in large cohorts of neonates admitted to NICUs in developing countries? To the best of our knowledge, this study represents the largest cohort to date that evaluates this issue in the developing world. As in most previous reports, BSI was the main cause of NI. In this study, BSI occurred at a rate of 12.0 per 1,000 patientdays and accounted for $16.3 \%$ of NIs. A higher proportion
(17.3 per 1,000 CVC-days) of all BSIs could be linked etiologically to a CVC. Other authors have reported a BSI incidence between $15.6 \%$ and $32.6 \%$. In our study, the rate of ventilator-associated pneumonia (VAP) was 3.2 per 1,000 ventilator-days. According to the NHSN, median VAP rates are 2.0 per 1,000 ventilator-days 9 . Because many NHSN hospitals use only microbiological reports to identify patients with VAP, 
TABLE 3 - Selected antimicrobial susceptibility patterns of isolates.

\begin{tabular}{|c|c|c|c|}
\hline \multirow[b]{2}{*}{ Microorganism/antimicrobial } & \multirow[b]{2}{*}{ Isolates tested (n) } & \multicolumn{2}{|c|}{ Resistance } \\
\hline & & $\mathrm{n}$ & $\%$ \\
\hline CoNS/oxacillin & 421 & 364 & 86.4 \\
\hline Staphylococcus aureus/oxacillin & 184 & 52 & 28.3 \\
\hline Staphylococcus aureus/vancomycin & 184 & 8 & 4.3 \\
\hline Enterococcus/vancomycin & 31 & 1 & 3.2 \\
\hline Enterobacter/third-generation cephalosporins & 42 & 9 & 21.4 \\
\hline Escherichia coli/third-generation cephalosporins & 58 & 5 & 8.6 \\
\hline Klebsiella pneumoniae/third-generation cephalosporins* & 57 & 18 & 31.6 \\
\hline Serratia marcescens/third-generation cephalosporins* & 36 & 13 & 36.1 \\
\hline Acinetobacter/third-generation cephalosporins* & 61 & 19 & 31.1 \\
\hline Pseudomonas aeruginosa/carbapenems $\dagger$ & 52 & 8 & 15.4 \\
\hline Pseudomonas aeruginosa/quinolones $¥$ & 52 & 8 & 15.4 \\
\hline Pseudomonas aeruginosa/ceftazidime & 52 & 8 & 15.4 \\
\hline
\end{tabular}

CoNS: coagulase-negative staphylococci; *Ceftriaxone, cefotaxime, or ceftazidime; †Imipenem or meropenem; ¥quinolones: ciprofloxacin.

NHSN rates may underestimate the true incidence of VAP. Therefore, the current VAP rate is probably more representative, with all diagnoses of VAP confirmed based on the review of patient medical records and microorganisms recovered from tracheal aspirates on the day of diagnosis.

Gram-negative rods are major pathogens of neonatal sepsis in developing countries ${ }^{10}$. In NICUs, following the adoption of sophisticated tertiary neonatal care with a high rate of invasive device use, CoNS stand out as the main agents of neonatal nosocomial sepsis, with Staphylococcus epidermidis the most frequently isolated ${ }^{11}$. Neonatal infection by CoNS is less severe but causes significant morbidity especially among infants of very low birth weight. In this study, CoNS was the most common microorganism causing NIs with microbiological diagnoses. These results are in strong agreement with other studies, similar to previous studies conducted in the unit ${ }^{12,13}$.

During this study, we observed a gradual decrease in gramnegative and an increase in gram-positive bacterial infection rates. In particular, in 2004, there was a reduction in infection rates demonstrated by a study conducted in the NICU to evaluate the impact of the NICU building environment on the risk of NI by surveillance during a 4-year period during which the NICU was moved from an old building to a temporary unit and finally to a new and better-designed unit, all in the absence of a change in staffing ${ }^{14}$.

Most CoNS isolates recovered from infants in this study were resistant to oxacillin. Nevertheless, similar to findings reported by Celebi et al. ${ }^{1}$, clinical improvement was documented in more than half of the patients. The CoNS isolates represented true bacteremia, and all study patients met the clinical criteria for sepsis. One possible explanation is that CoNS is a low-virulence pathogen. According to Ben Jaballah et al.$^{15}$, high resistance to third-generation cephalosporin by gram-negative bacilli has increased, including Acinetobacter sp, Enterobacter sp, Serratia marcescens, Klebsiella pneumoniae, and Pseudomonas aeruginosa. This was shown in some of our studies including detection of outbreaks of these pathogens ${ }^{16-18}$.

The most important NI remains BSI with staphylococci as the predominant pathogens, which showed much higher rates than those reported in the literature ${ }^{19}$. Multiresistant microorganisms, especially oxacillin-resistant staphylococci and gram-negative bacilli resistant to cephalosporin were frequently found. The process of evaluating the causative organisms was valuable in itself by helping to draw the attention of health care professionals to this important cause of morbidity and by promoting the use of strict hygiene measures and meticulous care of the infected infants.

\section{CONFLICT OF INTEREST}

The authors declare that there is no conflict of interest.

\section{FINANCIAL SUPPORT}

This research was supported by the Federal University of Uberlândia, MG, Brazil.

\section{REFERENCES}

1. Celebi S, Hacimustafaoglu M, Ozdemir O, Ozakin C. Nosocomial Gram-positive bacterial infections in children: results of a 7 year study. Pediatr Int 2007; 49:875-882.

2. Hocevar SN, Edwards JR, Horan TC, Morrell GC, Iwamoto M, Lessa FC. Device-associated infections among neonatal intensive care unit patients: 
incidence and associated pathogens reported to the National Healthcare Safety Network, 2006-2008. Infec Control Hosp Epidemiol 2012; 33:1200-1206.

3. Srivastava S, Shetty N. Healthcare-associated infections in neonatal units: lesson from contrasting worlds. J Hosp Infect 2007; 65:292-306.

4. National Nosocomial Infections Surveillance System (NNIS). NNIS system report, data summary from January 1992 through June 2004, issue October 2004. Am J Infect Control 2004; 32:470-485.

5. Center for Diseases Control and Prevention (CDC). The National Healthcare Safety Network (NHSN) manual Patient Safety Component Protocol. Division of Healthcare Quality Promotion. Atlanta, GA, USA: CDC; 2008

6. Yalaz M, Altun-Köroglu O, Ulusoy B, Yildiz B, Akisu M, Vardar F, et al. Evaluation of device-associated infections in a neonatal intensive care unit. Turk J Pediatr 2012; 54:128-135.

7. Couto RC, Carvalho EAA, Pedrosa TMG, Pedroso ER, Mozar Neto C, Biscione FM. A 10-year prospective surveillance of nosocomial infections in neonatal intensive care units. Am J Infect Control 2007; 35:183-189.

8. Chitnis AS, Magill SS, Edwards JR, Chiller TM, Fridkin SK, Lessa FC. Trends in Candida central line-associated bloodstream infections among NICUs, 1999-2009. Pediatrics 2012; 130:46-52.

9. Rosenthal VD, Rodríguez-Calderón ME, Rodríguez-Ferrer M, Singhal T, Pawar M, Sobreyra-Oropeza M, et al. Findings of the international nosocomial infection control consortium (INICC), Part II: impact of a multidimensional strategy to reduce ventilator-associated pneumonia in neonatal intensive care units in 10 developing countries. Infect Control Hosp Epidemiol 2012; 33:704-710.

10. Macharashvili N, Kourbatova E, Butsashvili M, Tsertsvadze T, McNutt LA, Leonard MK. Etiology of neonatal blood stream infections in Tbilisi, Republic of Georgia. Int J Infect Dis 2009; 13:499-505.

11. Jean-Baptiste N, Benjamin Jr DK, Cohen-Wolkowiez M, Cohen-Wolkowiez M, Fowler Jr VG, Laughon M, et al. Coagulase-negative staphylococcal infections in the neonatal intensive care unit. Infect Control Hosp Epidemiol $2011 ; 32: 679-686$.

12. Brito DVD, von Dolinger EJO, Abdallah VOS, Darini ALC, Gontijo Fo PP. Two outbreaks of mixed etiology associated with central venous catheters inserted by phlebotomy in critical neonates. Braz J Infect Dis 2009; 13:177-182.

13. Brito CS, Brito DVD, Abdallah VOS, Gontijo Filho PP. An outbreak of sepsis associated/related with the use of central venous catheter in a Neonatal Intensive Care Unit. Rev Panam Infectol 2009; 11:15-17.

14. Brito DVD, Silva HA, Oliveira EJ, Arantes A, Abdallah VOS, Tannus Jorge M, et al. Effect of neonatal intensive care unit environment on the incidence of hospital-acquired infection in neonates. J Hosp Infect 2007; 65:314-318

15. Ben Jaballah N, Bouziri A, Mnif Khaled, Hamdi A, Khladi A, Kchaou W. Epidemiology of hospital-acquired bloodstream infections in a Tunisian pediatric intensive care unit: a 2-year prospective study. Am J Infect Control 2007; 35:613-618.

16. Brito DVD, Matos C, Abdallah VOS, Diogo Filho A, Gontijo Fo PP. An outbreak of nosocomial infection caused by ESBLs producing Serratia marcescens in a Brazilian neonatal unit. Braz J Infect Dis 1999; 3:149-155.

17. Brito DVD, Oliveira EJ, Darini ALC, Abdallah VOS, Gontijo Fo PP. Nosocomial outbreaks due to Pseudomonas aeruginosa and Acinetobacter baumannii in a Neonatal Intensive Care Unit (NICU) of the Uberlândia Federal University Hospital. Braz J Microbiol 2003; 34 (supl I):27-28.

18. Brito DVD, Oliveira EJ, Matos C, Abdallah VOS, Gontijo Fo PP. An outbreak of conjunctivitis caused by multiresistant Pseudomonas aeruginosa in a Brazilian Newborn Intensive Care Unit. Braz J Infect Dis $2003 ; 7: 234-235$.

19. van der Zwet WC, Kaiser AM, van Elburg RM, Berkhof J, Fetter WP, Parlevliet GA, et al. Nosocomial infections in a Dutch neonatal intensive care unit: surveillance study with definitions for infection specifically adapted for neonates. J Hosp Infect 2005; 61:300-311. 